\title{
Cholesterol Oxidation Control in Mayonnaise Using Aframomum danielli Antioxidant Extracts
}

\author{
Etti, C.J ${ }^{1}$, Adegoke, G. O ${ }^{2}$, Etti, I. C ${ }^{3}$ \\ ${ }^{1}$ Department of Agricultural and Food Engineering, University of Uyo, Akwa Ibom State, Nigeria \\ ${ }^{2}$ Department of Food Technology, University of Ibadan, Nigeria \\ ${ }^{3}$ Department of Pharmacology and Toxicology, University of Uyo, Akwa Ibom State, Nigeria
}

\begin{abstract}
A study was carried out to monitor the control of cholesterol oxidation in mayonnaise using Aframomum danielli (A. danielli) antioxidant extracts. Mayonnaise samples were treated with A. danielli antioxidant extracts from diethyl ether, ethanol, n-hexane and that of a synthetic antioxidant butylated hydroxyl anisole (BHA) and these samples were assessed for control of cholesterol oxidation. The yield of A. danielli antioxidant extracts from the different solvents was highest in diethyl ether solvent (13.07\%), followed by the yield from ethanol solvent $(12.90 \%)$ while $n$-hexane solvent gave the lowest yield $(10.95 \%)$. Using high performance liquid chromatography (HPLC), the percentage cholesterol anti-oxidation effectiveness in mayonnaise indicated that Aframomum danielli antioxidant extracts at 200 part per million (ppm) concentration from the solvents diethyl ether, ethanol and $\mathrm{n}$-hexane were $93.96 \%, 99.54 \%$ and $94.94 \%$ respectively compared to $82.00 \%$ of synthetic antioxidant butylated hydroxyl anisole (BHA) on the $60^{\text {th }}$ day of mayonnaise storage, making Aframomum danielli antioxidant extracts to be better than synthetic antioxidant extracts BHA in the control of cholesterol oxidation in mayonnaise.
\end{abstract}

Key words: Antioxidant extracts, Aframomum danielli, Cholesterol oxidation, Mayonnaise, BHA

\section{INTRODUCTION}

Cholesterol is a molecule with a double bond in its structure and is therefore susceptible to oxidation leading to the formation of oxysterols which could result in some cytotoxic, mutagenic, atherogenic and carcinogenic effects on human consumption [1]. Foods that are naturally characterized by high cholesterol contents are major sources of oxysterols when processed, such as eggs and egg-derived products which mayonnaise is one of them. Oxidation of cholesterol may be initiated by poly-unsaturated fatty acid [2]. Interaction of triglycerides with cholesterol may accelerate the oxidation of the sterol and cholesterol may also influence triglyceride oxidation [3]. Oxygen free radicals and hydroxyl free radicals, superoxides and hydrogen peroxide formed from the reaction may induce cholesterol oxidation [4].

In the light of the potentially dangerous effects of oxysterols on human health, efforts to prevent or to reduce oxysterol consumption are now currently being made. Anti-oxidants of both synthetic and natural origins are widely applied to prevent lipids and cholesterol oxidation in processed foods, raw materials or fats and oils used in manufacturing of various foods [5]. However, antioxidant action is directed to prevent or delay fatty acid oxidation. The effects of antioxidants to prevent or control oxysterols in processed foods are currently not being investigated enough.

Antioxidants are organic lipid or water soluble substances that can scavenge the active forms of oxygen involved in the initiation steps of oxidation, or break the oxidative chain reaction. Antioxidants may react with the fatty acid peroxy radicals to form stable antioxidant radicals, which are either insufficiently reactive for further reactions or form non radical products [5]. Synthetic antioxidants have been used for a long time although some concerns have arisen due to the possible potential toxicity in some [6]. Natural antioxidants, which are present at variable amounts in vegetables such as fruits, leaves, flowers, roots, grains and seeds have gained prominence as alternatives to synthetic antioxidants [7],[8].

The natural spice Aframomum danielli (A. danielli) is known to possess preservative properties [9]. It is rich in nutrients and its antioxidant potential is better than synthetic antioxidants like butylated hydroxytoulene (BHT) and butylated hydroxyanisole (BHA) [10];[11]. It has a potent synergistic inhibitory effects on food spoilage yeast when used in combination with hydrostatic pressure [12]. The preservative capability of the powder of $A$. danielli has been associated with phytochemical components tentatively identified as alkaloids [13]. This work is aimed at using a natural antioxidant $A$. danielli in controlling cholesterol oxidation in mayonnaise. 


\section{MATERIALS AND METHODS}

\subsection{Extraction of antioxidant extracts:}

The fresh pods of A. danielli (obtained from Bode Market, Oyo state of Nigeria) were sun dried for five days before removing from the seeds. The seeds were sorted to remove extraneous materials and winnowed to remove adhering particles and dirts. The seeds of A. danielli were air dried at $27 \pm 2^{\circ} \mathrm{C}$ for 3 days due to its low moisture of about $10.9 \%$ determined by the method[14]. The seeds were then pulverized into a tiny powdery form in a hammer mill after drying and it was stored at $4^{\circ} \mathrm{C}$ until it was used.

\subsection{Solvent extraction of $A$. danielli spice:}

The method described by [15] was used for solvent extraction of $A$. danielli spice. 500ml each of diethyl ether solvent, ethanol solvent and $\mathrm{n}$-hexane solvent were used to extract finely ground A. danielli spice under refluxing condition for 10 hours respectively. The filtrate of each extraction was freed of the solvent to recover the extract by evaporation of the solvent. The filtrate which was the spice extract was packaged and kept at $4^{\circ} \mathrm{C}$ until it was used.

\subsection{Production of Mayonnaise:}

This was done according to the method described by [16]. 10.80g of egg yolk was mixed thoroughly with dissolved salt $1.50 \mathrm{~g}$, Sugar $2.50 \mathrm{~g}$, cinnamon $0.20 \mathrm{~g}$ and vinegar $10.80 \mathrm{~g}$ for about $2-3$ minutes in a laboratory mixer ( Kenwood Cheff model). Oil (soybean oil) was slowly added to the mixture to form an emulsion as the mixing proceeded. The addition of oil lasted for about 15 minutes. 10-15\% of the oil was added slowly during the first five minutes of the mixing and 50\% of the oil was added during the next five minutes of the mixing and then the remaining $35-40 \%$ of the oil was added gradually during the last five minutes of the mixing. After the production of the mayonnaise, it was packaged in transparent plastic materials, sealed and stored at room temperature.

\subsection{Antioxidant Incorporation:}

The antioxidant extract of A. danielli spice extracted with diethyl ether, ethanol and $\mathrm{n}$-hexane solvents were incorporated into different samples of prepared mayonnaise at $200 \mathrm{ppm}$ concentration by direct addition using the method of [17]. Synthetic antioxidant BHA was also incorporated into another mayonnaise sample at 200ppm concentration also using [17] method. Control sample which was the sample with no antioxidant extract was also obtained. Chemical tests were carried out on the five samples of mayonnaise.

\subsection{Extraction of Organic phase:}

This was determined by the method described by [18]. $2.0 \mathrm{~g}$ of mayonnaise was weighed into $250 \mathrm{ml}$ flask. $25 \mathrm{ml}$ of alcoholic potassium hydroxide solution ( 0.5 molar in $95 \%$ ethanol) was added and boiled gently under reflux for one hour. The saponified sample solution was transferred to a separating funnel using $50 \mathrm{ml}$ of water to wash the flask. The solution was extracted while warm 3 times with $50 \mathrm{ml}$ quantities of diethyl ether. Each extracts was poured into another separator containing $20 \mathrm{ml}$ of water. After the third extract was added, the mixture of the ether extracts with $20 \mathrm{ml}$ of water was shaken vigorously with two other $20 \mathrm{ml}$ quantities of water respectively. The ether extracts was washed with $20 \mathrm{ml}$ of aqueous 0.5 molar potassium hydroxide solution and twice with $20 \mathrm{ml}$ quantities of water until washed water was no longer alkaline to phenolphthalein. The ether extract was poured into a weighed flask and the solvent was evaporated off and the residue dried at about $80^{\circ} \mathrm{C}$ and weighed to constant weight. The organic phase was re-dissolved with ethanol before injection into a HPLC system.

\subsection{Equipment:}

Cholesterol analyses were performed by Cecil CE 1200 HPLC equipped with UV detector and a Cecil injection valve with $20 \mu$ l Loop. The separation was carried out on a C18 Zorbax 3000SB column $250 \mathrm{~mm} \times$ $4.6 \mathrm{~mm}$ internal diameter (I.D) of $5 \mu \mathrm{m}$ particle size with $100 \%$ of $\mathrm{MeOH}$ as mobile phase at a flow rate $1.0 \mathrm{~mL} / \mathrm{min}$ at ambient temperature $\left(27^{\circ} \mathrm{C}\right)$ and $8 \mathrm{~min}$ running time. The chromatograms were processed at wavelengths of maximum absorption $\mu \mathrm{V}(215 \mathrm{~nm})$. The identification of cholesterol was by noting the retention time $\left(t_{R}\right)$, maximum absorption of each samples, maximum absorption of standard sample and their dilution factors (d.f). hence, the following formulae were used to determine the cholesterol in each samples.

$$
\% \text { Cholesterol in solution }=\frac{\lambda \max ^{\mathrm{b}}}{\lambda \max ^{\mathrm{a}}} \times \mathrm{A}_{3} \times d . f
$$

Where; $\lambda \mathrm{max}^{\mathrm{b}}$, was the maximum absorption of the samples, $\lambda \max ^{\mathrm{a}}$ was the maximum absorption of the standard sample $(\mathrm{nm}), \mathrm{A}_{3}$ was concentration of the standard sample in $\%$ and $d . f$ was the dilution factors of the samples. 


\subsection{Determination of Cholesterol antioxidant effectiveness:}

The percentage cholesterol antioxidant effectiveness was done using the method described by [11]

$$
\text { CHE }=\frac{\text { cholesterol of control sample -cholesterol of test sample }}{\text { cholesterol of control sample }} \times 100
$$

Where; $C H E$ was cholesterol antioxidant effectiveness.

III. Result and Discussion:

\begin{tabular}{|c|c|c|c|c|}
\hline solvent & $\begin{array}{l}\text { Vol. of } \\
\text { solvent used } \\
(\mathrm{ml})\end{array}$ & \begin{tabular}{|l} 
Quantity of \\
A. danielli \\
powder used (g)
\end{tabular} & $\begin{array}{l}\text { Yields of } A \text {. } \\
\text { Danielli extracts (g) }\end{array}$ & $\begin{array}{l}\text { (\%) yield of } A . \\
\text { danielli extracts }\end{array}$ \\
\hline Diethyl ether & 500 & 85 & 11.11 & 13.07 \\
\hline Ethanol & 500 & 92 & 11.87 & 1290 \\
\hline n-hexane & 500 & 85 & 9.31 & $10.95^{-}$ \\
\hline
\end{tabular}

Table 2: HPLC quantification of cholesterol oxidation level of mayonnaise using Retention time ( $\mathrm{t}$ ), highest

\begin{tabular}{|c|c|c|c|c|}
\hline Sample No. & $t_{R}(\min )$ & $2 m a x^{j}(\mathrm{~nm})$ & $\begin{array}{l}\text { Dilution } \\
\text { factor (d.f) }\end{array}$ & $\begin{array}{l}\% \text { cholesterol in } \\
\text { solution }\end{array}$ \\
\hline Chl3010 & $7: 32.4$ & 778019.81 & $\times 10$ & 0.22 \\
\hline Chl2020 & $7=05.2$ & 25504210.00 & $\times 12$ & 8.61 \\
\hline Chl2030 & $7=28.0$ & 10284380.00 & $\times 10$ & 2.89 \\
\hline $\mathrm{Chl} 2040$ & $7=29.6$ & 17016784.00 & $\times 100$ & 47.84 \\
\hline Ch13020 & $7: 24.8$ & 8608772.00 & $\times 10$ & 2.42 \\
\hline Chl3000 & $7=09.1$ & 3554491.00 & & \\
\hline
\end{tabular}

*Presented according to chromatogram shown in figures 1 - 6 obtained with PAD-HPLC system in methanol

$*_{t_{R}}$ is the retention time and $\lambda m c x x^{3}$ is the maximum absorption of samples.

Key:

Chl3010: sample with ethanol antioxidant extracts

Ch12020: sample with synthetic antioxidant BHA

Chl2030: sample with diethyl ether antioxidant extracts

Ch12040: control sample with no antioxidant

Chl3020: sample with n-hexane antioxidant extracts

Chl3000: Standard (0.1\%)

Table 3: Cholesterol oxidation levels in mayonnaise treated with antioxidant extracts of A. danielli using HPLC

\begin{tabular}{lll}
\hline Sample No. & Cholesterol in solution \% & \% control of cholesterol oxidation \\
\hline Chl3010 & 0.22 & 99.54 \\
Chl2020 & 8.61 & 82.00 \\
Chl2030 & 2.89 & 93.96 \\
Chl3020 & 2.42 & 94.94 \\
Chl2040 & 47.84 & Control \\
\hline
\end{tabular}

The yield of the antioxidant extracts from A. danielli spice is shown in table 1 . The result showed that diethyl ether solvent gave the highest extracts yield amongst the three solvents while $\mathrm{n}$-hexane solvent yielded the lowest. Polarity of solvents appear to affect the yield of extraction according to [15] and it was confirmed in this study that the polar the solvent, the higher the yield of extraction.
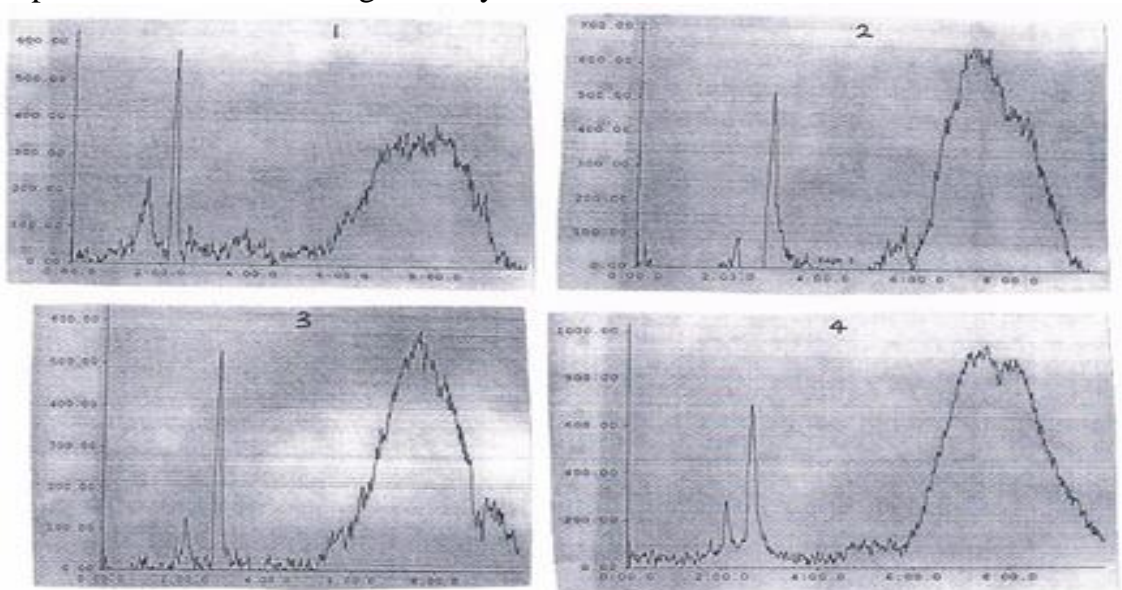

Figures 1, 2, 3, and 4: Chromatogram profile of mayonnaise samples Chl3010, Chl2020, Chl2030 and Chl2040 respectively 
Mayonnaise treated with A. danielli extracts from ethanol, diethyl ether and n-hexane at 200ppm concentration gave higher percentage antioxidant effectiveness of $99.54 \%, 93.95 \%$ and $94.94 \%$ respectively compared to the sample with synthetic antioxidant (BHA) with $82.00 \%$ of cholesterol antioxidant effectiveness as shown in table 3. Figures 1, 2, 3, 4, 5 and 6 show the chromatogram profile of the mayonnaise samples. The characterization of the peaks and their area which are represented by maximum wavelength of absorption are presented in table 2. Ethanol extracts of the antioxidant was the best effective in the control of cholesterol oxidation in the mayonnaise with $0.22 \%$ cholesterol in solution at 7:32.4 retention time and $778019.81 \mathrm{~nm}$ absorption with the dilution factor $(\times 10)$ compared to synthetic antioxidant (BHA) which controlled the cholesterol oxidation to $8.61 \%$ at 7:05.2 retention time and $25504210.00 \mathrm{~nm}$ absorption with $(\times 12)$ dilution factor. The control sample (Chl2040) exhibited a great deal of cholesterol oxidation of 47.84\% at 7:29.6 retention time and $17016784.00 \mathrm{~nm}$ absorption with $(\times 100)$ dilution factor. The dilution factor of the cholesterol sample was very high $(\times 100)$ in order to enable the chromatogram profile of the control sample to be seen clearly with the HPLC system.

Generally, according to [1], natural antioxidants show inhibitory action to cholesterol oxidation and $A$. danielli is a natural antioxidant. Also, [19] reported that natural antioxidants have several advantages over synthetic antioxidants such as; natural antioxidants are readily acceptable by consumers as they are considered to be safe, no safety tests might be required by legislation for they belong to a component of food that are generally regarded as safe.

\section{CONCLUSION}

The result of the study showed that A. danielli being a natural antioxidant can be used to control cholesterol oxidation in mayonnaise product at $200 \mathrm{ppm}$. The result also showed that $A$. danielli has a better efficiency than synthetic antioxidant BHA in mayonnaise in terms of controlling cholesterol oxidation. However, further research is necessary in order to investigate the active component in A. danielli that is responsible for the cholesterol anti-oxidation in mayonnaise

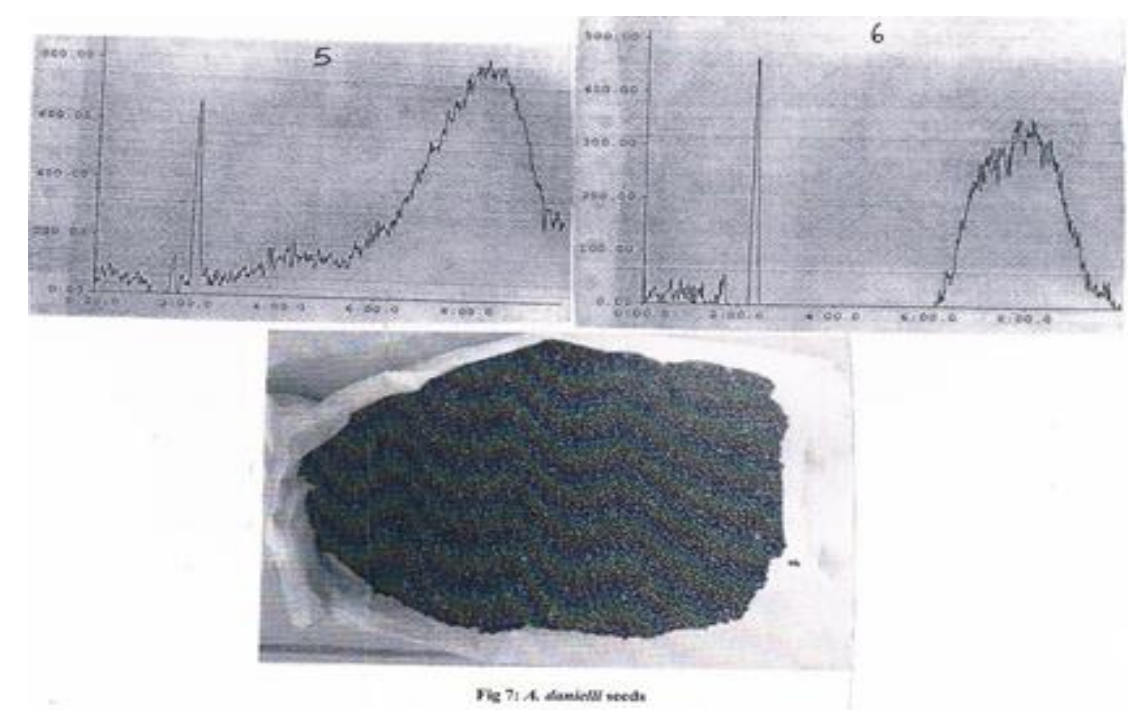

Figures 5, 6 and 7: Chromatogram profile of mayonnaise samples Chl3020, Chl3000 and A.danielli seeds respectively

\section{REFERENCES}

[1] A. Valenzuela, J. Sanhueza, and S. Nieto, "Natural antioxidants in functional foods: from food safety to health benefits Grasas and Aceites in Press," 2003.

[2] S. Kubow, "Lipid oxidation products in food and atherogenesis," Nut. Rev., vol. 51, pp. 33-40, 1993.

[3] S. K. Kim and W. W. Nawar, "Oxidative interactions of cholesterol with triglycerides," J. Am. Oil Chem.Soc., vol. 68, pp. 931-934, 1991.

[4] J. H. Lee, D. W. Shoeman, S. S. Kim, and A. S. Csallany, "The effects of superoxide anion in the production of seven major cholesterol oxidation products in aprotic and protic conditions," Int. J. Food Sci. Nutr, vol. 48, pp. 151-159, 1977.

[5] A. Valenzuela and S. Nieto, "Natural and synthetic antioxidants: Food quality protectors," Grasas and Aceites, vol. 47, pp. 186-196, 1996. 
[6] P. A. Lachance, B. S. Zeina, and W. S. Jeong, "Antioxidants: an integrative approach," Nutrition, vol. 17, pp. 835-842, 2001.

[7] O. I. Aruoma, "Extracts as antioxidant prophylactic agents," INFORM, vol. 8, pp. 1236-1242, 1997.

[8] O. I. Aruoma et al., "An evaluation of the antioxidant and antiviral action of extracts of rosemary and proven cal herbs," Food Chem Toxical, vol. 34, no. 5, pp. 449-56, 1996.

[9] G. O. Adegoke et al., "Protection of maize (Zeamays) and soybean (Glycin max) using Afamomum danielli," Eur. Food Res. Technol., vol. 214, pp. 408-411, 2002.

[10] G. O. Adegoke and B. J. Skura, "Nutritional profile and antioxidant spectrum of the spice Aframomum danielli," Plant Food Human Nut., vol. 45, pp. 175-182, 1994.

[11] G. O. Adegoke and A. G. Gopalakrishna, "Extraction and identification of antioxidants from the spice Aframomum danielli,” J. Amer. Oil. Chem. Soc., vol. 75, pp. 1047-1052, 1998.

[12] G. O. Adegoke, H. Iwahashi, and Y. Komatsu, "Inhibition of saccharomyces cerevisae by combination of hydrostatic pressure and monoterpenes," J. Food Sci, vol. 62, pp. 404-405, 1997.

[13] G. O. Adegoke, S. B. Fasoyiro, and B. J. Skura, "Control of microbial growth browning and lipid oxidation by spice Aframomum danielli," Eur. Food Res. Technol., vol. 211, pp. 342-345, 2001.

[14] AOAC, Official Methods of Analysis (14th ed). Association of Official Analytical Chemists, Washington D. C, 14th ed. 1984.

[15] S. S. Chang, M. B. Ostic, O. Q. Hseih, and C. L. Huang, "Natural antioxidant from rosemary and sage," J. Food Sci, vol. 42, pp. 1102-1106, 1977.

[16] J. W. Corrant, "Emulsion Technology and History of Mayonnaaise," Chem. Publ, vol. 50, 2003.

[17] J. Smith, "Food additives users handbook Glasgow: Academic Press," pp. 5-46, 1991.

[18] D. Pearson, The Chemical analysis of foods. New York: Chemical Publishing Company, 1991.

[19] J. Pokorny, "Natural antioxidants for food use," Trends Food Sci. Technol., vol. 2, pp. 223-227, 1991. 